\title{
SEMIDIRECT PRODUCTS OF (TOPOLOGICAL) SEMI-ABELIAN ALGEBRAS
}

\author{
MARIA MANUEL CLEMENTINO, ANDREA MONTOLI, AND LURDES SOUSA
}

\begin{abstract}
We give a description of the semidirect products in any semi-abelian variety. Moreover, we internalize this description to the models of semi-abelian theories in any finitely complete category. As an application, we characterize the topology of the semidirect products in the topological models of any semi-abelian theory.
\end{abstract}

\section{INTRODUCTION}

The semidirect product is a classical construction in group theory, which is used to obtain an equivalence between group actions and split extensions. D. Bourn and G. Janelidze gave in [6] a categorical definition of semidirect products, and proved that it still gives an equivalence between split extensions and internal actions (in the sense of [4]) in the context of what were later called semi-abelian categories [10], i.e. pointed Barr-exact protomodular categories with finite colimits.

In the category of groups, the categorical semidirect product coincides with the classical one. Moreover, it is known that the semidirect product of two groups, with respect to a given action, is, as a set, the cartesian product of the two groups. This is not true in all semi-abelian varieties. E.B. Inyangala proved in [9] that it is true in varieties of right $\Omega$-loops, by constructing, given two right $\Omega$-loops $X$ and $B$ and an action $\xi$ of $B$ on $X$, bijections $\varphi$ and $\psi$, inverse to each other, which are morphisms of split extensions (in the category of pointed sets), as in the following diagram

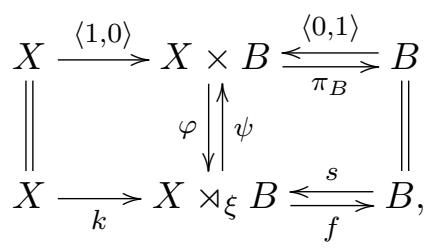

where the bottom row is the split extension corresponding to $\xi$. (A similar result was first obtained in [2], where varieties of right $\Omega$-loops are called pointed semi-associative Mal'tsev varieties.) Moreover, in [9] it was shown that, for a pointed variety with two binary operations, the set maps $\varphi$ and $\psi$ may yet be defined, and, whenever they are inverse to each other, the variety is always one of $\Omega$-loops. J.R.A. Gray and N. Martins-Ferreira showed in [8] that varieties of right $\Omega$-loops are indeed the unique pointed varieties where there are natural bijections $\varphi$ and $\psi$ as above.

Furthermore, F. Borceux and M.M. Clementino proved in [3] that the equivalence between internal actions and split extensions, obtained via the categorical semidirect product, holds in the categories of topological models of any semi-abelian algebraic theory.

In the present paper, we give a description of the semidirect products in any semi-abelian variety, showing that the semidirect product corresponding to an internal action of an object $B$ on an object $X$ can be described as a subset of the cartesian product of $B$ and a suitable number of copies of $X$, reinterpreting a result of [8]. Moreover, we use this fact to prove that, in the case of topological models of a semi-abelian theory, the semidirect product is always a retract of the 
topological product of $B$ and some copies of $X$.

The paper is organized as follows: in Section 2 we recall the categorical definition of semidirect product. In Section 3 we describe semidirect products in the context of semi-abelian varieties, showing that a semidirect product of two objects $X$ and $B$ as above can be always seen as a subset of the cartesian product of $B$ and $X^{n}$ for some natural number $n$ (depending only on the operations in the variety). In Section 4 we characterize the semi-abelian varieties in which the inclusion of the semidirect product into the corresponding cartesian product is a bijection, showing that any semi-abelian variety with this property is a variety of right $\Omega$-loops; in this way we generalize Inyangala's results. Moreover, we study an example of a variety that can be described as semi-abelian using two different sets of operations, with different cardinality, showing that they give rise to different inclusions of the semidirect products into the corresponding cartesian products. In Section 5 we study explicitly other concrete examples. In Section 6 we internalize the description of the semidirect products, obtained in the previous sections, to the models of semi-abelian theories in any finitely complete category. In particular, we consider the case of topological models of semi-abelian theories, and we describe the topology of the semidirect product in this context.

\section{The CATEgorical notion of SEMidirect PROduCt}

In this section we recall from [6] the categorical notion of semidirect product.

Let $\mathbb{C}$ be a finitely complete category. For any morphism $p: E \rightarrow B$ in $\mathbb{C}$, we can define the pullback functor

$$
p^{*}: \operatorname{Pt}(B) \rightarrow \operatorname{Pt}(E),
$$

where the category $\operatorname{Pt}(B)$, called the category of points over $B$, is the category of points of the comma category $\mathbb{C}$ over $B$, i.e. the cocomma category $1_{B}$ over $\mathbb{C} / B$. This amounts to the category whose objects are the split epimorphisms with codomain $B$. In fact a morphism from the terminal object $1_{B}: B \rightarrow B$ to an object $f: A \rightarrow B$ is precisely an arrow $s: B \rightarrow A$ such that $f s=1_{B}$.

Definition 2.1. A finitely complete category $\mathbb{C}$ is said to be a category with semidirect products if, for any arrow $p: E \rightarrow B$ in $\mathbb{C}$, the pullback functor $p^{*}$ (has a left adjoint and) is monadic.

In this case, denoting by $T^{p}$ the monad defined by this adjunction, given a $T^{p}$-algebra $(D, \xi)$ the semidirect product $(D, \xi) \rtimes(B, p)$ is an object in $\operatorname{Pt}(B)$ corresponding to $(D, \xi)$ via the canonical equivalence $K$ :

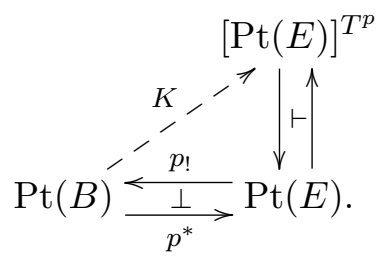

Let us recall from [6] that, being $\mathbb{C}$ finitely complete, the pullback functors $p^{*}$ have left

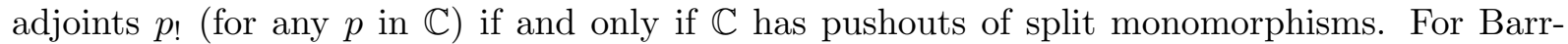
exact categories [1], if, moreover, the functors $p^{*}$ are conservative, that is if $\mathbb{C}$ is protomodular [5], the existence of semidirect products is guaranteed. In fact:

Theorem 2.2 ([6], Theorem 3.4). A finitely complete Barr-exact category is a category with semidirect products if and only if it is protomodular and has pushouts of split monomorphisms.

If $\mathbb{C}$ is finitely complete, so that we can define $p^{*}$ for every morphism $p$, has pushouts of split monomorphisms, so that the functors $p^{*}$ have left adjoints $p_{!}$, and an initial object 0 , then it is enough to consider the functors $i_{B}{ }^{*}$ for the unique morphisms $i_{B}: 0 \rightarrow B$ :

Proposition 2.3 ([13], Corollary 3). Let $\mathbb{C}$ be a category with finite limits, pushouts of split monomorphisms and initial object. Then the following statements are equivalent: 
(i) all pullback functors $i_{B}^{*}$ defined by the initial arrows are monadic;

(ii) for any morphism $p$ in $\mathbb{C}$, the pullback functor $p^{*}$ is monadic, i.e. $\mathbb{C}$ admits semidirect products.

When the category $\mathbb{C}$ is pointed, the algebras for the monad $\left(T^{i_{B}}, \eta, \mu\right)$ are called internal actions in [4] and the endofunctor $T^{i_{B}}$ is usually denoted by $B b(-)$. We recall that $\eta_{X}$ and $\mu_{X}$ are the unique morphisms such that $k_{0} \eta_{X}=\iota_{X}$ and $k_{0} \mu_{X}=\left[k_{0}, \iota_{B}\right] k_{0}^{\prime}$, as displayed in the diagrams

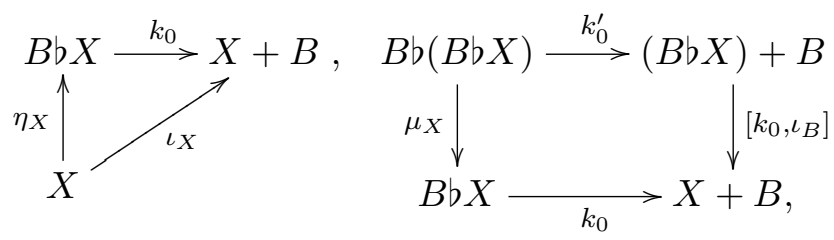

where $k_{0}^{\prime}$ and $k_{0}$ denote the kernels of $[0,1]:(B b X)+B \rightarrow B$ and of $[0,1]: X+B \rightarrow B$, respectively.

The algebras for this monad are pairs $(X, \xi: B b X \rightarrow X)$ satisfying the usual conditions:

$$
\xi \eta_{X}=1_{X} \quad \text { and } \quad \xi \mu_{X}=\xi(1 b \xi) .
$$

Consequently, for $\mathbb{C}$ as above, saying that $\mathbb{C}$ has semidirect products means that, for each internal action $\xi: B b X \rightarrow X$, there exists a unique (up to isomorphism) split epimorphism $A \underset{f}{\stackrel{s}{\longrightarrow}} B$ such that $X=\operatorname{Ker} f$ and making the following diagram commute (see [12] for more details):

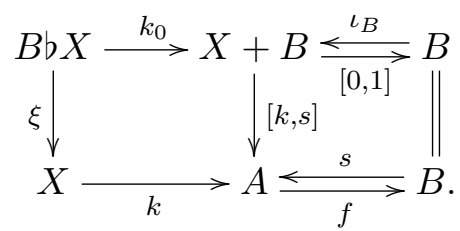

Then $A \underset{f}{\stackrel{s}{\longrightarrow}} B$ is the semidirect product of $X$ and $B$ with respect to $\xi$. Sometimes we will identify this semidirect product with the object $A$ or with the split extension

$$
X \stackrel{k}{\longrightarrow} A \underset{f}{\stackrel{s}{\longrightarrow}} B .
$$

When $\mathbb{C}$ is the category of groups, $B b X$ is the subgroup of the free product $X+B$ generated by the elements of the form $b x b^{-1}$, with $b \in B$ and $x \in X$. Hence an internal action $\xi$ is completely determined by $\xi\left(b x b^{-1}\right)=s(b) k(x) s(b)^{-1}$, and so $\xi$ is the realization in $X$ of the conjugation in the classical semidirect product $X \rtimes_{\xi} B$.

\section{Semidirect Products in SEmi-Abelian varieties}

An immediate consequence of Theorem 2.2 is that every semi-abelian category [10] has semidirect products in the categorical sense recalled above. This is the case, in particular, of semiabelian varieties, which were characterized by D. Bourn and G. Janelidze in [7].

Theorem 3.1. A variety of universal algebra is semi-abelian if and only if it has, among its operations, a unique constant $0, n$ binary operations $\alpha_{i}, i=1, \ldots, n$, and an $(n+1)$-ary operation $\theta$ satisfying the following equations:

$$
\begin{array}{cc}
\alpha_{i}(x, x) & =0 \quad \text { for any } x \\
\theta(\underline{\alpha}(x, y), y) & =x \quad \text { for any } x, y,
\end{array}
$$

where $\underline{\alpha}(x, y)$ denotes $\left(\alpha_{1}(x, y), \ldots, \alpha_{n}(x, y)\right)$. 
The aim of this section is to give a description of the semidirect product in semi-abelian varieties.

We start by recalling a result of E.B. Inyangala [9]. Let $\mathbb{C}$ be a variety of right $\Omega$-loops, i.e. a (semi-abelian) variety which has, among its operations, a unique constant 0 , a binary + and a binary - satisfying the following equations:

(a) $x+0=x$

(b) $0+x=x$;

(c) $(x-y)+y=x$;

(d) $(x+y)-y=x$.

Then, given a split extension $(* *)$ in $\mathbb{C}$, it is possible to define two set maps

$$
\begin{aligned}
& \varphi: X \times B \longrightarrow A \quad \psi: A \longrightarrow X \times B \\
& (x, b) \longmapsto x+s(b) \quad a \longmapsto(a-s f(a), f(a)),
\end{aligned}
$$

treating $k$ as an inclusion.

Proposition $3.2([9])$. The two maps $\varphi$ and $\psi$ are inverse to each other.

Therefore, given any split extension (**), $A$ is bijective to the cartesian product of $B$ and $\operatorname{Ker} f$.

Moreover, E.B. Inyangala proved in [9] that, if a semi-abelian variety $\mathbb{C}$ has a binary + and a binary -, and the maps $\varphi$ and $\psi$ are defined using + and - as above, then $\varphi$ and $\psi$ are bijections inverse to each other, whose restrictions to $X$ and $B$ are identities, if and only if the equations (a)-(d) are satisfied, i.e. if and only if $\mathbb{C}$ is a variety of right $\Omega$-loops. Later, in [8] J.R.A. Gray and N. Martins-Ferreira extended this result, showing that such maps $\varphi$ and $\psi$ are components of natural isomorphisms between suitable functors, and, conversely, the existence of natural isomorphisms between those functors induces binary operations + and - making the algebras right $\Omega$-loops.

We are now going to express the natural transformations studied in [8] in terms of the operations of the semi-abelian variety, in order to get a description of semidirect products. This way we will obtain some results already contained in [8], but we will give different proofs, that will be useful later in the study of semidirect products of topological algebras.

Let $\mathbb{C}$ be a semi-abelian variety. For each split extension $(* *)$ in $\mathbb{C}$, using the operations $\alpha_{1}, \ldots, \alpha_{n}$ and $\theta$ introduced at the beginning of the section, we can define two set maps

$$
\begin{aligned}
\varphi: X^{n} \times B & \longrightarrow A & \psi: A & \longrightarrow X^{n} \times B \\
(\underline{x}, b) & \longmapsto \theta(\underline{x}, s(b)) & a & \longmapsto
\end{aligned}
$$

where $\underline{x}$ denotes $\left(x_{1}, \ldots, x_{n}\right)$, and $k$ is treated again as an inclusion. To simplify our calculations, for any map $h: Z \rightarrow W$ and $\underline{z}=\left(z_{1}, \ldots, z_{n}\right) \in Z^{n}, h^{n}(\underline{z})$ denotes $\left(h\left(z_{1}\right), \ldots, h\left(z_{n}\right)\right)$.

Proposition 3.3. For any split epimorphism $A \underset{f}{\stackrel{s}{\longrightarrow}} B$ in $\mathbb{C}$, we have:

1. $\varphi \psi=1_{A}$, and therefore $A$ is a retract of $X^{n} \times B$.

2. $A$ is in bijection with the subset

$$
Y=\left\{(\underline{x}, b) \in X^{n} \times B \mid \underline{\alpha}(\theta(\underline{x}, s(b)), s(b))=\underline{x}\right\} .
$$

Proof. 1. For any $a \in A$ we have:

$$
\varphi \psi(a)=\varphi(\underline{\alpha}(a, s f(a)), f(a))=\theta(\underline{\alpha}(a, s f(a)), s f(a))=a,
$$

where the last equality follows from equation (II).

2. Let us first prove that $\psi(A) \subseteq Y$ : for any $a \in A$, we have $\psi(a)=(\underline{\alpha}(a, s f(a)), f(a))$, hence:

$$
\underline{\alpha}(\theta(\underline{\alpha}(a, s f(a)), s f(a)), s f(a))=\underline{\alpha}(a, s f(a)) .
$$


It remains to prove that $\psi \varphi_{\left.\right|_{Y}}=1_{Y}$. Let us observe that, for any algebra $Z$ and any $z \in Z$, we have $\theta(\underline{0}, z)=\theta(\underline{\alpha}(z, z), z)=z$. Then, for any $(\underline{x}, b) \in Y$ :

$$
\begin{aligned}
\psi \varphi(\underline{x}, b) & =\psi \theta(\underline{x}, s(b)) \\
& =(\underline{\alpha}(\theta(\underline{x}, s(b)), s f \theta(\underline{x}, s(b))), f \theta(\underline{x}, s(b))) \\
& =\left(\underline{\alpha}\left(\theta(\underline{x}, s(b)), \theta\left(s^{n} f^{n}(\underline{x}), s f s(b)\right)\right), \theta\left(f^{n}(\underline{x}), f s(b)\right)\right) \\
& =(\underline{\alpha}(\theta(\underline{x}, s(b)), \theta(\underline{0}, s(b))), \theta(\underline{0}, b)) \\
& =(\underline{\alpha}(\theta(\underline{x}, s(b)), s(b)), b)=(\underline{x}, b),
\end{aligned}
$$

where the last equality holds because $(\underline{x}, b) \in Y$.

Proposition 3.3 allows us to give a description of semidirect products in any semi-abelian variety.

Theorem 3.4. Given a semi-abelian variety $\mathbb{C}$ and a split epimorphism $A \underset{f}{\stackrel{s}{\leftrightarrows}} B$ in $\mathbb{C}$, let $X$ be a kernel of $f$ and $\xi: B b X \rightarrow X$ the corresponding internal action of $B$ on $X$. Then the semidirect product $X \rtimes_{\xi} B$ of $X$ and $B$ w.r.t. the action $\xi$ is the set $Y$ described in the previous proposition:

$$
Y=\left\{(\underline{x}, b) \in X^{n} \times B \mid \underline{\alpha}(\theta(\underline{x}, s(b)), s(b))=\underline{x}\right\},
$$

equipped with the following structure: if $\omega$ is an m-ary operation of the variety, then in $Y$ we have:

$$
\omega_{Y}\left(\left(\underline{x}_{1}, b_{1}\right), \ldots,\left(\underline{x}_{m}, b_{m}\right)\right)=\left(\xi^{n} \underline{\alpha}_{B b X}\left(\omega_{B b X}\left(\theta_{B b X}\left(\underline{x}_{1}, b_{1}\right), \ldots, \theta_{B b X}\left(\underline{x}_{m}, b_{m}\right)\right), \omega_{B b X}(\underline{b})\right), \omega_{B}(\underline{b})\right) .
$$

Proof. Diagram $(*)$ says that $\xi$ is the restriction of the morphism $[k, s]$. We know that $A$ is bijective to $Y$ via the maps $\varphi$ and $\psi$ studied in Proposition 3.3. Given $\left(\underline{x}_{i}, b_{i}\right) \in Y$, for $i=1, \ldots, m$, let

Then

$$
u=\omega_{A}\left(\theta_{A}\left(\underline{x}_{1}, s\left(b_{1}\right)\right), \ldots, \theta_{A}\left(\underline{x}_{m}, s\left(b_{m}\right)\right)\right) .
$$

$$
\begin{aligned}
\omega_{Y}\left(\left(\underline{x}_{1}, b_{1}\right), \ldots,\left(\underline{x}_{m}, b_{m}\right)\right) & =\psi \omega_{A}\left(\varphi\left(\underline{x}_{1}, b_{1}\right), \ldots, \varphi\left(\underline{x}_{m}, b_{m}\right)\right)=\psi(u) \\
& =\left(\underline{\alpha}_{A}(u, s f(u)), f(u)\right) .
\end{aligned}
$$

Since $f$ and $s$ are morphisms (and so they preserve the operations):

$$
f(u)=\omega_{B}\left(\theta_{B}\left(f^{n}\left(\underline{x}_{1}\right), f s\left(b_{1}\right)\right), \ldots, \theta_{B}\left(f^{n}\left(\underline{x}_{m}\right), f s\left(b_{m}\right)\right)\right),
$$

and, since $f s=1_{B}$ and $X=\operatorname{Ker} f$,

$$
f(u)=\omega_{B}\left(\theta_{B}\left(\underline{0}, b_{1}\right), \ldots, \theta_{B}\left(\underline{0}, b_{m}\right)\right)=\omega_{B}(\underline{b}) .
$$

Thus, $s f(u)=\omega_{A}\left(s^{m}(\underline{b})\right)$, and we obtain:

$$
\begin{aligned}
\omega_{Y}\left(\left(\underline{x}_{1}, b_{1}\right), \ldots,\left(\underline{x}_{m}, b_{m}\right)\right)=\left(\underline{\alpha}_{A}\left(u, \omega_{A}\left(s^{m}(\underline{b})\right)\right), \omega_{B}(\underline{b})\right) \\
=\left(\underline{\alpha}_{A}\left(\omega_{A}\left(\theta_{A}\left(\underline{x}_{1}, s\left(b_{1}\right)\right), \ldots, \theta_{A}\left(\underline{x}_{m}, s\left(b_{m}\right)\right)\right), \omega_{A}\left(s^{m}(\underline{b})\right)\right), \omega_{B}(\underline{b})\right) \\
=\left([k, s]^{n} \underline{\alpha}_{B b X}\left(\omega_{B b X}\left(\theta_{B b X}\left(\underline{x}_{1}, b_{1}\right), \ldots, \theta_{B b X}\left(\underline{x}_{m}, b_{m}\right)\right), \omega_{B b X}(\underline{b})\right), \omega_{B}(\underline{b})\right),
\end{aligned}
$$

and, since $\xi$ is the restriction of $[k, s]$, we finally obtain the claimed equality.

\section{A Detailed Description of $\varphi$ AND $\psi$}

The aim of this section is to make explicit the relationships between the properties of the maps $\varphi$ and $\psi$ defined in Section 3 and the equations of the variety.

Let $\mathbb{C}$ be a variety which has, among its operations, a unique constant $0, n$ binary operations $\alpha_{i}, i=1, \ldots, n$, satisfying the equations (I), and an $(n+1)$-ary operation $\theta$. Given a split epimorphism with kernel $X$ as in (**), let $\varphi: X^{n} \times B \rightarrow A$ and $\psi: A \rightarrow X^{n} \times B$ be the maps 
defined in Section 3. Observe that the map $\psi$ is well-defined (i.e. it takes value in $X^{n} \times B$ ) thanks to the equations (I). We have therefore the following diagram:

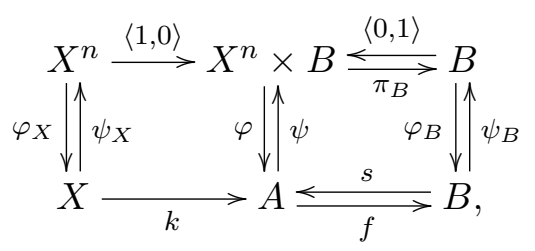

where $\varphi_{X}, \psi_{X}$ are obtained via the universal property of kernels, and

$$
\varphi_{B}(b)=f(\varphi(\underline{0}, b))=f \theta(\underline{0}, s(b))=\theta(\underline{0}, b), \quad \psi_{B}(b)=\pi_{B}(\psi(s(b)))=1_{B},
$$

so that $f \varphi=\varphi_{B} \pi_{B}$ and $\psi s=\langle 0,1\rangle \psi_{B}$.

The following proposition is a reformulation of some results in [8].

Proposition 4.1. We have that:

1. $\varphi_{B}=1_{B}$ for any split epimorphism (**) if and only if the following equation is satisfied in the variety:

$$
\theta(\underline{0}, x)=x \quad \text { for all } x
$$

2. $\varphi \psi=1_{A}$ for any split epimorphism (**) if and only if equation (II) is satisfied in the variety;

3. if equation (III) holds in the variety, then $\psi \varphi=1_{X^{n} \times B}$ for any split epimorphism (**) if and only if the equation

$$
\underline{\alpha}(\theta(\underline{x}, y), y)=\underline{x} \quad \text { for all } x, y
$$

is satisfied in the variety.

Proof. 1. Suppose that equation (III) holds. Then $\varphi_{B}(b)=\theta(\underline{0}, b)=b$, for all $b \in B$. Conversely, suppose that $\varphi_{B}=1_{B}$ for any split epimorphism (**). Applying this fact to the split epimorphism $A \underset{1_{A}}{\stackrel{1_{A}}{\longrightarrow}} A$ for any algebra $A$, we easily get equation (III).

2. The fact that equation (II) implies that $\varphi \psi=1_{A}$ was already proved (see Proposition 3.3 ). Conversely, suppose that $\varphi \psi=1_{A}$ for any split epimorphism of the form $A \times A \stackrel{\langle 1,1\rangle}{\longleftarrow} A$. Then, for any $x, y \in A$, we have

$$
(x, y)=\theta(\underline{\alpha}((x, y),(y, y)),(y, y))=(\theta(\underline{\alpha}(x, y), y), \theta(\underline{\alpha}(y, y), y)),
$$

and hence $\theta(\underline{\alpha}(x, y), y)=x$ for all $x, y$.

3. We have that

$$
\begin{aligned}
\psi \varphi(\underline{x}, b) & =\psi(\theta(\underline{x}, s(b))=(\underline{\alpha}(\theta(\underline{x}, s(b)), s f \theta(\underline{x}, s(b))), f \theta(\underline{x}, s(b))) \\
& =\left(\underline{\alpha}\left(\theta(\underline{x}, s(b)), \theta\left(s^{n} f^{n}(\underline{x}), s f s(b)\right)\right), \theta\left(f^{n}(\underline{x}), f s(b)\right)\right) \\
& =(\underline{\alpha}(\theta(\underline{x}, s(b)), \theta(\underline{0}, s(b))), \theta(\underline{0}, b)) \\
& =(\underline{\alpha}(\theta(\underline{x}, s(b)), s(b)), b) .
\end{aligned}
$$

Hence, if equation (IV) holds, we get $\psi \varphi=1_{X^{n} \times B}$. Conversely, suppose that $\psi \varphi=1$ for any split epimorphism of the form $A \times A \underset{\pi_{2}}{\stackrel{\langle 1,1\rangle}{\longrightarrow}} A$. Then, for any $\underline{x}, y$, we have

$$
(\underline{x}, y)=(\underline{\alpha}(\theta(\underline{x}, y), y), y)
$$

and the first component of this equality gives equation (IV). 
Theorem 4.2. 1 . For each semi-abelian variety $\mathbb{C}$, and each $n$ binary operations $\alpha_{i}$ and $(n+1)$ ary operation $\theta$ satisfying equations (I)-(II), for any split epimorphism $A \underset{f}{\stackrel{s}{\leftrightarrows}} B$ the maps $\varphi$ and $\psi$ are bijections between $A$ and the cartesian product $X^{n} \times B$ if and only if equation (IV) is satisfied in $\mathbb{C}$.

2. If a semi-abelian variety $\mathbb{C}$ satisfies equation (IV), then it is possible to define in $\mathbb{C}$ binary operations + and - satisfying the conditions for right $\Omega$-loops.

Proof. 1. Thanks to the previous proposition, it suffices to observe that equations (I) and (II) imply equation (III); indeed:

$$
\theta(\underline{0}, x)=\theta(\underline{\alpha}(x, x), x)=x .
$$

2. The operations + and - can be defined in the following way:

$$
x+y=\theta(\underline{\alpha}(x, 0), y), \quad x-y=\theta(\underline{\alpha}(x, y), 0) .
$$

They satisfy the equations of a right $\Omega$-loop:

(a) $x+0=\theta(\underline{\alpha}(x, 0), 0)=x$, by equation (II);

(b) $0+x=\theta(\underline{\alpha}(0,0), x)=\theta(\underline{0}, x)=x$;

(c)

$$
\begin{aligned}
(x-y)+y & =\theta(\underline{\alpha}(x, y), 0)+y=\theta(\underline{\alpha}(\theta(\underline{\alpha}(x, y), 0), 0), y) & & \text { (by (IV)) } \\
& =\theta(\underline{\alpha}(x, y), y)=x & & \text { (by (II)); }
\end{aligned}
$$

$$
\begin{aligned}
(x+y)-y & =\theta(\underline{\alpha}(x, 0), y)-y=\theta(\underline{\alpha}(\theta(\underline{\alpha}(x, 0), y), y), 0) & & (\text { by }(\mathrm{IV})) \\
& =\theta(\underline{\alpha}(x, 0), 0)=x & & (\text { by (II)). }
\end{aligned}
$$

Let us observe that J.R.A. Gray and N. Martins-Ferreira proved in [8] that the existence of suitable maps $\varphi$ and $\psi$ for any split epimorphism in a variety (giving natural transformations of suitable functors between categories of points) allows to define operations $\theta$ and $\alpha_{i}$ satisfying equations (I). This means that, in a semi-abelian variety, for any set of operations $\left(\alpha_{i}, \theta\right)$ there exists exactly one pair of maps $(\varphi, \psi)$ for any split epimorphism. Theorem 4.2 extends then the results of [9] and [8], giving a complete characterization of those semi-abelian varieties in which the semidirect product of two objects $X$ and $B$ (w.r.t. an action of $B$ on $X$ ) naturally underlies the cartesian product of $B$ and a certain number of copies of $X$, and, moreover, we show that one single copy of $X$ suffices.

Let us observe, moreover, that, if a semi-abelian variety $\mathbb{C}$ satisfies the conditions of Theorem 4.2, then the maps $\varphi$ and $\psi$ induce bijections between $X$ and $X^{n}$ for any split epimorphism (**). If $n \geq 2$, this implies that all the algebras of the variety, except the trivial one, are infinite. The following is an example of a variety, with $n \geq 2$, which satisfies equation (IV).

Example 4.3. Let $\mathbb{C}$ be the variety defined by the following operations: a unique constant 0 , two binary operations $\alpha_{1}$ and $\alpha_{2}$ and a ternary operation $\theta$ satisfying the equations (I), (II) and (IV). A concrete example of an algebra belonging to this variety is given by the set $\mathbb{R}^{\mathbb{N}}$ of real sequences (but $\mathbb{R}$ can be replaced by any non-trivial, not necessarily infinite, right $\Omega$-loop) equipped with the operations defined by

$$
\begin{gathered}
\alpha_{1}(x, y)=\left(x_{2 n-1}-y_{2 n-1}\right)_{n \in \mathbb{N}}=\left(x_{1}-y_{1}, x_{3}-y_{3}, \ldots\right), \\
\alpha_{2}(x, y)=\left(x_{2 n}-y_{2 n}\right)_{n \in \mathbb{N}}=\left(x_{2}-y_{2}, x_{4}-y_{4}, \ldots\right), \\
\theta(x, y, z)=\left(x_{1}+z_{1}, y_{1}+z_{2}, x_{2}+z_{3}, y_{2}+z_{4}, \ldots\right),
\end{gathered}
$$


for any $x=\left(x_{n}\right)_{n \in \mathbb{N}}, y=\left(y_{n}\right)_{n \in \mathbb{N}}$ and $z=\left(z_{n}\right)_{n \in \mathbb{N}}$ in $\mathbb{R}^{\mathbb{N}}$. It is immediate to see that the equations (I) are satisfied. Concerning equation (II) we have:

$$
\begin{aligned}
\theta\left(\alpha_{1}(x, y), \alpha_{2}(x, y), y\right) & =\theta\left(\left(x_{2 n-1}-y_{2 n-1}\right)_{n \in \mathbb{N}},\left(x_{2 n}-y_{2 n}\right)_{n \in \mathbb{N}}, y\right) \\
& =\left(x_{1}-y_{1}+y_{1}, x_{2}-y_{2}+y_{2}, x_{3}-y_{3}+y_{3}, x_{4}-y_{4}+y_{4}, \ldots\right) \\
& =x .
\end{aligned}
$$

Finally, concerning equation (IV), we have:

$$
\begin{aligned}
\alpha_{1}(\theta(x, y, z), z) & =\alpha_{1}\left(\left(x_{1}+z_{1}, y_{1}+z_{2}, x_{2}+z_{3}, y_{2}+z_{4}, \ldots\right), z\right) \\
& =\left(x_{1}+z_{1}-z_{1}, x_{2}+z_{3}-z_{3}, \ldots\right)=x
\end{aligned}
$$

and

$$
\begin{aligned}
\alpha_{2}(\theta(x, y, z), z) & =\alpha_{2}\left(\left(x_{1}+z_{1}, y_{1}+z_{2}, x_{2}+z_{3}, y_{2}+z_{4}, \ldots\right), z\right) \\
& =\left(y_{1}+z_{2}-z_{2}, y_{2}+z_{4}-z_{4}, \ldots\right)=y
\end{aligned}
$$

Then $\mathbb{C}$ satisfies the conditions of Theorem 4.2 , and hence, for any split extension $(* *)$ in $\mathbb{C}$, we have that $A$ is bijective to the cartesian product $X^{2} \times B$. This example can be easily generalized to the case of any $n \geq 2$. Observe that, in this case, the operations + and - which give the structure of right $\Omega$-loop are the usual sum and subtraction of sequences, respectively, i.e.:

$$
\begin{aligned}
& x+y=\left(x_{n}+y_{n}\right)_{n \in \mathbb{N}}, \\
& x-y=\left(x_{n}-y_{n}\right)_{n \in \mathbb{N}} .
\end{aligned}
$$

Then $\mathbb{C}$ can be seen as a semi-abelian variety using both sets of operations $\{0,+,-\}$ and $\left\{0, \alpha_{1}, \alpha_{2}, \theta\right\}$. Using the first one, we have that, for any split extension (**), the object $A$ is bijective to $X \times B$, while, using the second one, $A$ is bijective to $X^{2} \times B$.

The previous example shows that, if a variety can be described as semi-abelian using two different sets of operations, then the two corresponding descriptions of the semidirect products may be different.

\section{EXAMPLES}

In this section we present examples that illustrate the results of Section 3 in the absence of equation (IV). Given a split epimorphism $A \underset{f}{\stackrel{s}{\leftrightarrows}} B$ in a semi-abelian variety $\mathbb{C}$ with binary operations $\alpha_{i}, i=1, \ldots, n$, and an $(n+1)$-operation $\theta$ satisfying equations (I) and (II), diagram $(* * *)$ :

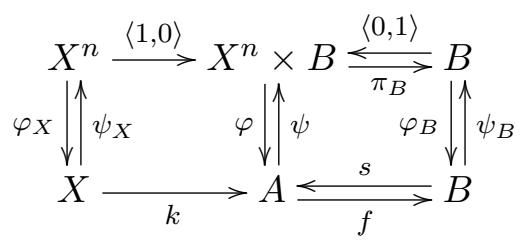

gives an inclusion $\langle 1,0\rangle \psi_{X}$ of $X$ into the cartesian product $X^{n} \times B$. In the following examples we show that this inclusion can be of different forms.

Example 5.1. Let $\mathbb{C}$ be the variety of Heyting semilattices, which is defined by a constant $T$ and two binary operations $\wedge$ and $\Rightarrow$ satisfying the following equations:

$$
\begin{array}{cl}
\top \wedge x=x, \quad x \wedge x=x, & x \wedge y=y \wedge x, \\
x \wedge(y \wedge z)=(x \wedge y) \wedge z, \quad & (x \Rightarrow x)=\top, \\
x \wedge(x \Rightarrow y)=x \wedge y, \quad y \wedge(x \Rightarrow y)=y, \\
x \Rightarrow(y \wedge z)=(x \Rightarrow y) \wedge(x \Rightarrow z) .
\end{array}
$$

P.T. Johnstone proved in [11] that the variety of Heyting semilattices is semi-abelian, with the following operations:

$$
\alpha_{1}(x, y)=(x \Rightarrow y), \quad \alpha_{2}(x, y)=(((x \Rightarrow y) \Rightarrow y) \Rightarrow x)
$$




$$
\theta(x, y, z)=(x \Rightarrow z) \wedge y \text {. }
$$

In this variety, given a split extension $(* *)$, we have, for $x \in X$ :

$$
\begin{aligned}
\psi(x)= & \left(\alpha_{1}(x, s f(x)), \alpha_{2}(x, s f(x)), f(x)\right)=\left(\alpha_{1}(x, \top), \alpha_{2}(x, \top), \top\right)= \\
= & ((x \Rightarrow \top),(((x \Rightarrow \top) \Rightarrow \top) \Rightarrow x), \top)=(\top, x, \top),
\end{aligned}
$$

hence the inclusion of $X$ into $X \times X \times B$ is given by the second inclusion of $X$ into the product:

$$
X \stackrel{\langle 0,1,0\rangle}{\longrightarrow} X \times X \times B .
$$

Example 5.2. Let $\mathbb{C}$ be the variety defined by the following operations: a unique constant 0 , a binary subtraction $\alpha$ (which means a binary operation $\alpha$ such that $\alpha(x, x)=0$ and $\alpha(x, 0)=x$ for any $x$ ) and a ternary operation $\theta$ satisfying the following equation:

$$
\theta(\alpha(x, y), \alpha(x, y), y)=x \text {. }
$$

It is a semi-abelian variety, with $n=2$ and $\alpha_{1}=\alpha_{2}=\alpha$. A concrete example of this situation is given by the divisible abelian group $(\mathbb{Q},+)$ with $\alpha$ and $\theta$ given by:

$$
\alpha(x, y)=x-y, \quad \theta(x, y, z)=\frac{x+y+2 z}{2} .
$$

In this variety, given a split extension $(* *)$, we have, for $x \in X$ :

$$
\psi(x)=(\alpha(x, s f(x)), \alpha(x, s f(x)), f(x))=(\alpha(x, 0), \alpha(x, 0), 0)=(x, x, 0),
$$

hence the inclusion of $X$ into $X \times X \times B$ is given by the diagonal of $X$ :

$$
X \stackrel{\langle 1,1,0\rangle}{\longrightarrow} X \times X \times B .
$$

Example 5.3. Let $\mathbb{C}$ be the semi-abelian variety having a unique constant 0 , a binary subtraction $\alpha$, a binary sum $\rho$ and a ternary operation $\theta$, such that $\rho$ and $\alpha$ satisfy the usual group equations, and, moreover, the following equation is satisfied:

$$
\theta(\alpha(x, y), \alpha(x, y), y)=x .
$$

A concrete example of this situation is the divisible abelian group $(\mathbb{Q},+)$ considered in the example above, with $\rho, \alpha$ and $\theta$ given by:

$$
\rho(x, y)=x+y, \quad \alpha(x, y)=x-y, \quad \theta(x, y, z)=\frac{x+y+2 z}{2} .
$$

There are two ways of describing $\mathbb{C}$ as a semi-abelian variety. One is with $n=1$, using the group operations $\rho$ and $\alpha$. From this point of view, given a split extension $(* *)$, we have that $A$ is bijective to the cartesian product $X \times B$. The second way is with $n=2$, using $\alpha_{1}=\alpha_{2}=\alpha$ and the ternary operation $\theta$. From this second point of view, given a split extension $(* *)$, we have that $A$ is bijective to a subset of the cartesian product $X \times X \times B$. The two points of view are not in contradiction, because, as observed in the previous example, the fact that $\alpha$ is a subtraction implies that the inclusion of $X$ into $X \times X \times B$ is given by the diagonal of $X$.

\section{The SEMIDIRECT PRODUCT OF TOPOLOGICAL ALGEBRAS}

F. Borceux and M.M. Clementino proved in [3] that, given a semi-abelian algebraic theory $\mathbb{T}$, the category $\operatorname{Top}(\mathbb{T})$ of its topological models has semidirect products in the categorical sense, although it is not a semi-abelian category, because it fails to be Barr-exact in general. The results presented in Section 3 allow us to give a description of the semidirect products in Top $(\mathbb{T})$, as we are going to show.

Let $\mathbb{T}$ be a semi-abelian theory determined by a constant $0, n$ binary operations $\alpha_{i}$ and an $(n+1)$-ary operation $\theta$ satisfying equations (I) and (II), and let $\mathcal{E}$ be a finitely complete category. We will denote by $\mathcal{E}(\mathbb{T})$ the category of models of $\mathbb{T}$ in $\mathcal{E}$. When $\mathcal{E}=\operatorname{Set}, \mathcal{E}(\mathbb{T})$ is the semi-abelian variety corresponding to the theory $\mathbb{T}$. Let

$$
U: \mathcal{E}(\mathbb{T}) \rightarrow \mathcal{E}
$$


be the (forgetful) functor which forgets the algebraic structure of any object in $\mathcal{E}(\mathbb{T})$. Given a split epimorphism $A \underset{f}{\stackrel{s}{\leftrightarrows}} B$ in $\mathcal{E}(\mathbb{T})$, we can construct internally in $\mathcal{E}$ the maps $\varphi$ and $\psi$ studied in Proposition 3.3, obtaining thus two morphisms in $\mathcal{E}$ :

$$
\varphi: U(X)^{n} \times U(B) \rightarrow U(A), \quad \psi: U(A) \rightarrow U(X)^{n} \times U(B),
$$

where $X$ is the kernel of $f$. The proof of Proposition 3.3 uses only finite limits, hence it is invariant under the Yoneda embedding. This means that, for any finitely complete category $\mathcal{E}$ and for any split extension (**) in $\mathcal{E}(\mathbb{T}), U(A)$ is a subobject of $U(X)^{n} \times U(B)$ (it is the subobject defined by a suitable equalizer, which internalizes the description of $Y$ given in Proposition 3.3, see Theorem 6.1 below). The same is true for the proofs of Proposition 4.1 and Theorem 4.2. Hence we get the following

Theorem 6.1. Let $\mathbb{T}$ be a semi-abelian algebraic theory, and $\mathcal{E}$ a finitely complete category. Given a split epimorphism $A \underset{f}{\stackrel{s}{\leftrightarrows}} B$ in $\mathcal{E}(\mathbb{T})$ with kernel $X$, the object $U(A) \in \mathcal{E}$ is isomorphic to the subobject of $U(X)^{n} \times U(B)$ given by the equalizer (in $\mathcal{E}$ ) of the morphisms

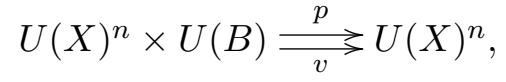

where $p$ is the first projection and $v$ is the morphism given by

$$
v=\left\langle\alpha_{1}, \ldots, \alpha_{n}\right\rangle \circ(\theta \times 1) \circ\left(U(k)^{n} \times U(s) \times U(s)\right) \circ\left(1 \times \Delta_{U(B)}\right) .
$$

If $\mathbb{T}$ is a variety of right $\Omega$-loops, then $U(A)$ is isomorphic to the product $U(X) \times U(B)$.

When $\mathbb{T}$ is a variety of right $\Omega$-loops, and $\mathcal{E}$ is a finitely complete category such that $\mathcal{E}(\mathbb{T})$ has pushouts of split monomorphisms, the previous theorem can be used to prove that the category $\mathcal{E}(\mathbb{T})$ has semidirect products in the categorical sense recalled in Section 2, as we show next.

Theorem 6.2. Let $\mathbb{T}$ be a theory of right $\Omega$-loops, and let $\mathcal{E}$ be a finitely complete category such that the category $\mathcal{E}(\mathbb{T})$ of models of $\mathbb{T}$ in $\mathcal{E}$ has pushouts of split monomorphisms. Then $\mathcal{E}(\mathbb{T})$ has semidirect products.

Proof. Since $\mathcal{E}(\mathbb{T})$ is pointed, finitely complete and has pushouts of split monomorphisms, thanks to Proposition 2.3 it suffices to consider only the pullback functors $i_{B}^{*}: \operatorname{Pt}(B) \rightarrow \operatorname{Pt}(0)=\mathcal{E}(\mathbb{T})$, for any $B \in \mathcal{E}(\mathbb{T})$. The category $\mathcal{E}(\mathbb{T})$ is protomodular, because $S e t(\mathbb{T})$ is, and the definition of a protomodular category is invariant under the Yoneda embedding. Hence, for any $B \in \mathcal{E}(\mathbb{T}), i_{B}^{*}$ is conservative. Moreover, it has a left adjoint, because $\mathcal{E}(\mathbb{T})$ has pushouts of split monomorphisms. To prove that $i_{B}^{*}$ is monadic, it suffices then to show that it creates coequalizers of parallel morphisms $g, h$ in $\operatorname{Pt}(B)$ such that $\bar{g}=i_{B}^{*}(g)$ and $\bar{h}=i_{B}^{*}(h)$ have an absolute coequalizer in $\mathcal{E}(\mathbb{T})$. Consider then the following diagram

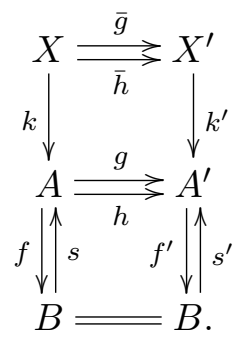

We know that, as objects of $\mathcal{E}, A$ and $A^{\prime}$ are isomorphic to $X \times B$ and $X^{\prime} \times B$, respectively. Moreover, the morphisms $g$ and $h$ are isomorphic (in $\mathcal{E}$ ) to $\bar{g} \times 1_{B}$ and $\bar{h} \times 1_{B}$, respectively, because the isomorphisms $\varphi$ and $\psi$ described before are natural. Consider then the following 
diagram in $\mathcal{E}$, where the upper line is an absolute coequalizer in $\mathcal{E}(\mathbb{T})$ :

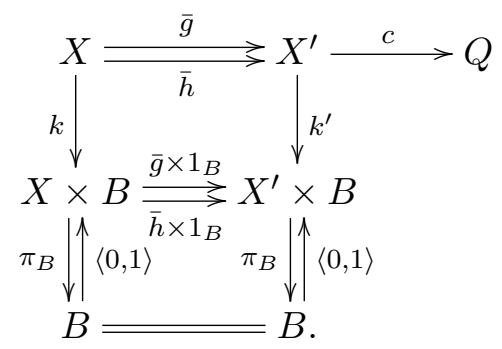

Since an absolute coequalizer is preserved by any functor, considering the functor $(-) \times B: \mathcal{E}(\mathbb{T}) \rightarrow \mathcal{E}$ we have that

$$
X \times B \underset{\bar{h} \times 1_{B}}{\stackrel{\bar{g} \times 1_{B}}{\longrightarrow}} X^{\prime} \times B \stackrel{c \times 1_{B}}{\longrightarrow} Q \times B
$$

is a coequalizer in $\mathcal{E}$. We now equip $Q \times B$ with a structure of an internal algebra. Let $\omega$ be an $m$-ary operation of the theory $\mathbb{T}$. Consider the following diagram:

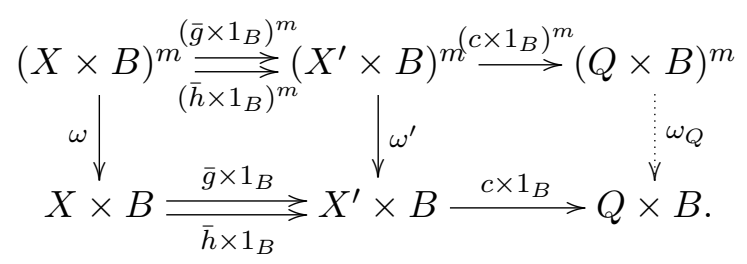

The upper row is still a coequalizer in $\mathcal{E}$. Its universal property defines the operation $\omega_{Q}$, since the two left hand side squares commute, being $\bar{g} \times 1_{B}$ and $\bar{h} \times 1_{B}$ morphisms in $\mathcal{E}(\mathbb{T})$. This operation $\omega_{Q}$ satisfies all the equations of the theory $\mathbb{T}$. Indeed, these equations can be expressed by diagrams in $\mathcal{E}$, and it can be shown that the ones concerning $Q \times B$ commute, because so do the ones concerning $A^{\prime} \cong X^{\prime} \times B$, using the fact that $c \times 1_{B}$ is an epimorphism in $\mathcal{E}(\mathbb{T})$ (because it is an epimorphism in $\mathcal{E}$ and the forgetful functor $\mathcal{E}(\mathbb{T}) \rightarrow \mathcal{E}$ is faithful). Hence $Q \times B$ is an object of $\mathcal{E}(\mathbb{T})$. It remains to prove that it is the coequalizer of $g=\bar{g} \times 1_{B}$ and $h=\bar{h} \times 1_{B}$ in $\mathcal{E}(\mathbb{T})$, and not only in $\mathcal{E}$ (the fact that it is preserved by $i_{B}^{*}$ is obvious). Consider the following diagram:

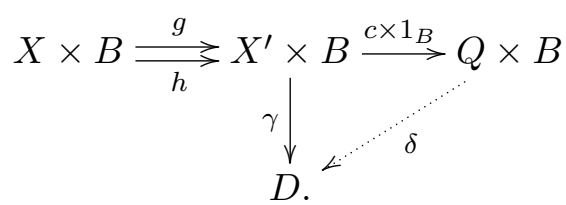

Since $c \times 1_{B}$ is the coequalizer of $g$ and $h$ in $\mathcal{E}$, if $\gamma g=\gamma h$ (where $\gamma$ is a morphism in $\mathcal{E}(\mathbb{T})$ ), there exists a unique morphism $\delta$ in $\mathcal{E}$ such that $\delta\left(c \times 1_{B}\right)=\gamma$. We only have to prove that $\delta$ is a morphism in $\mathcal{E}(\mathbb{T})$, i.e. that it preserves any operation $\omega$ of the theory $\mathbb{T}$. If $\omega$ is an $m$-ary operation, consider the following diagram:

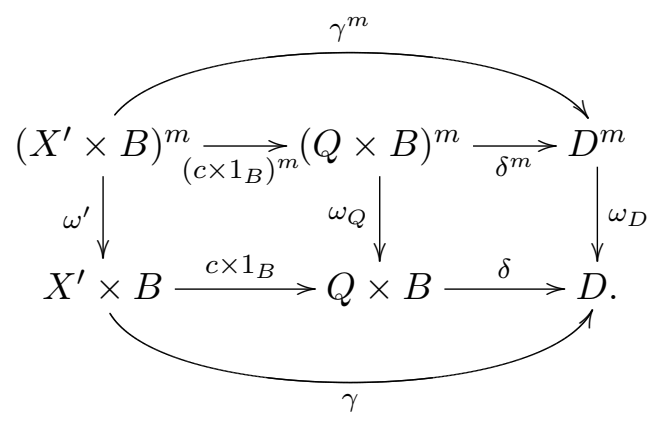

The square on the left and the whole rectangle commute, because $c \times 1_{B}$ and $\gamma$ are morphisms in $\mathcal{E}(\mathbb{T})$. Since $\left(c \times 1_{B}\right)^{m}$ is an epimorphism, the square on the right also commutes, and hence 
$\delta$ is a morphism in $\mathcal{E}(\mathbb{T})$. This concludes the proof, because the coequalizers in $\operatorname{Pt}(B)$ are built as in $\mathcal{E}(\mathbb{T})$.

We now consider the particular case when $\mathcal{E}=T o p$. If $\mathbb{T}$ is a semi-abelian algebraic theory, for any split extension $(* *)$ in $\operatorname{Top}(\mathbb{T})$ we have that, as a topological space, $A$ is a subspace, actually a retract, of the topological product $X^{n} \times B$. More explicitly:

Corollary 6.3. Given a semi-abelian theory $\mathbb{T}$ and a split extension $(* *)$ in $T o p(\mathbb{T})$, the maps

$$
\varphi: X^{n} \times B \rightarrow A \quad \text { and } \quad \psi: A \rightarrow X^{n} \times B
$$

of Proposition 3.3 are continuous.

Proof. $\varphi$ and $\psi$ are defined using only the operations $\theta$ and $\alpha_{i}$, that are continuous because they are morphisms in Top, and canonical morphisms induced by the products in Top, hence they are continuous.

Theorem 6.1 then particularizes to the following

Proposition 6.4. Let $\mathbb{T}$ be a semi-abelian algebraic theory. Given a split extension (**) in Top $(\mathbb{T})$, the algebra $A$ is homeomorphic to the set $Y \subseteq X^{n} \times B$ described in Proposition 3.3 equipped with the subspace topology of the product topology of $X^{n} \times B$. In particular, if the theory $\mathbb{T}$ defines a variety of right $\Omega$-loops, then $A$ is isomorphic, as a topological space, to the topological product $X \times B$.

We conclude by observing that the results of this section have the following interesting consequence. Let $\mathbb{T}$ be a theory of right $\Omega$-loops, and let $\mathcal{E}$ be a subcategory of $T o p$ which is closed under finite products (like the subcategories Comp, Haus, HComp, Conn and TotDisc of compact, Hausdorff, compact Hausdorff, connected, and totally disconnected spaces, respectively). Given a split extension $(* *)$ in $T o p(\mathbb{T})$, if both $X$ and $B$ belong to $\mathcal{E}$, then $A$ belongs to $\mathcal{E}$, too. This means that, if both $X$ and $B$ are compact, then $A$ also is, and the same holds for the properties of being Hausdorff, connected and totally disconnected. In particular, the subcategories $\operatorname{Haus}(\mathbb{T}), \operatorname{HComp}(\mathbb{T})$ and $\operatorname{Tot} \operatorname{Disc}(\mathbb{T})$, being closed under limits and finite coproducts in $\operatorname{Top}(\mathbb{T})$, have semidirect products, that coincide with the corresponding topological semidirect products.

\section{ACKNOWLEDGEMENTS}

We are very grateful to Francis Borceux for suggesting us the study of semidirect products in topological semi-abelian algebras and to George Janelidze for useful discussions on this topic.

This work was partially supported by the Centro de Matemática da Universidade de Coimbra (CMUC), funded by the European Regional Development Fund through the program COMPETE and by the Portuguese Government through the FCT - Fundação para a Ciência e a Tecnologia under the projects PEst-C/MAT/UI0324/2013 and MCANA PTDC/MAT/120222/2010 and grant number SFRH/BPD/69661/2010.

\section{REFERENCES}

[1] M. Barr, Exact categories, in: Lecture Notes in Mathematics, vol. 236 (1971), Springer-Verlag, 1-120.

[2] F. Borceux, D. Bourn, Mal'cev, protomodular, homological and semi-abelian categories, Mathematics and its applications, vol. 566 (2004), Kluwer.

[3] F. Borceux, M.M. Clementino, Topological semi-abelian algebras, Adv. Math. 190 (2005), no. 2, 425-453.

[4] F. Borceux, G. Janelidze, G.M. Kelly, Internal object actions, Commentationes Mathematicae Universitatis Carolinae 46 (2005), no. 2, 235-255.

[5] D. Bourn, Normalization equivalence, kernel equivalence and affine categories, in: Lecture Notes in Mathematics, vol. 1488 (1991), Springer-Verlag, 43-62.

[6] D. Bourn, G. Janelidze, Protomodularity, descent, and semidirect products, Theory Appl. Categories 4 (1998), 37-46.

[7] D. Bourn, G. Janelidze, Characterization of protomodular varieties of universal algebra, Theory Appl. Categories 11 (2003), 143-147. 
[8] J.R.A. Gray, N. Martins-Ferreira, On algebraic and more general categories whose split epimorphisms have underlying product projections, Appl. Categorical Structures, available online, DOI 10.1007/s10485-013-93365.

[9] E.B. Inyangala, Semidirect products and crossed modules in varieties of right $\Omega$-loops, Theory Appl. Categories 25 (2011), 426-435.

[10] G. Janelidze, L. Márki, W. Tholen, Semi-abelian categories, J. Pure Appl. Algebra 168 (2002), no. 2-3, 367-386.

[11] P.T. Johnstone, A note on the semiabelian variety of Heyting semilattices, Fields Institute Communications 43 (2004), 317-318.

[12] N. Martins-Ferreira, M. Sobral, On categories with semi-direct products, J. Pure Appl. Algebra 216 (2012), 1968-1975.

[13] G. Metere, A. Montoli, Semidirect products of internal groupoids, J. Pure Appl. Algebra 214 (2010), 1854-1861.

CMuC, Department of Mathematics, University of Coimbra, 3001-501 Coimbra, Portugal

E-mail address: mmc@mat.uc.pt

CMuC, Department of Mathematics, University of Coimbra, 3001-501 Coimbra, Portugal

E-mail address: montoli@mat.uc.pt

Polytechnic Institute of Viseu, Portugal \& CMUC, Centre for Mathematics of the University of Coimbra, Portugal

E-mail address: sousa@estv.ipv.pt 